\title{
Real-time inversion recovery for infarct visualization during MR-guided interventions
}

\author{
Adrienne E Campbell-Washburn*, Toby Rogers, Jonathan R Mazal, Michael S Hansen, Robert J Lederman, \\ Anthony Z Faranesh \\ From 19th Annual SCMR Scientific Sessions \\ Los Angeles, CA, USA. 27-30 January 2016
}

\section{Background}

Real-time MR imaging is appealing for dynamic procedural guidance. Some MRI-guided procedures, such as catheter ablation or myocardial biopsy, may require the interventionist to visualize infarcted tissue in real-time in order to navigate devices relative to the lesion. Methods to interleave late gadolinium enhancement (LGE) images into a real-time imaging stream ( 1 in 5 frames) have been described previously for infarct visualization (1). Here, we implemented a real-time inversion recovery sequence to provide a stream of LGE images for procedural guidance, designed to rapidly toggle between high-frame rate "navigation mode" and lower-frame rate "infarct visualization mode".

\section{Methods}

Gadopenetate dimeglumine (Bayer Healthcare, Wayne, New Jersey) was administered intravenously $(0.2 \mathrm{mmol} /$ $\mathrm{kg}$ ) to a swine model of myocardial infarction. Standard real-time imaging was used for "navigation mode" (bSSFP, $\mathrm{TE} / \mathrm{TR}=1.27 / 2.54 \mathrm{~ms}$, flip angle $=45^{\circ}, \mathrm{FOV}=300 \mathrm{~mm}$, slice thickness $=6 \mathrm{~mm}$, matrix $=192 \times 144$, GRAPPA factor $=2$ ). In "infarct visualization mode", a non-selective inversion pre-pulse was performed before each real-time bSSFP acquisition. Inversion time (TI) was implemented as a real-time interactive parameter to enable optimal myocardial nulling, typical TI $=320 \mathrm{~ms}$ (approximately 25 minutes post-injection). The next inversion pulse immediately followed the image acquisition, with no additional time for signal recovery. A checkbox was used to switch between imaging modes on the fly.

Cardiovascular and Pulmonary Branch, Division of Intramural Research, National Heart, Lung, and Blood Institute, National Institutes of Health, Bethesda, MD, USA 

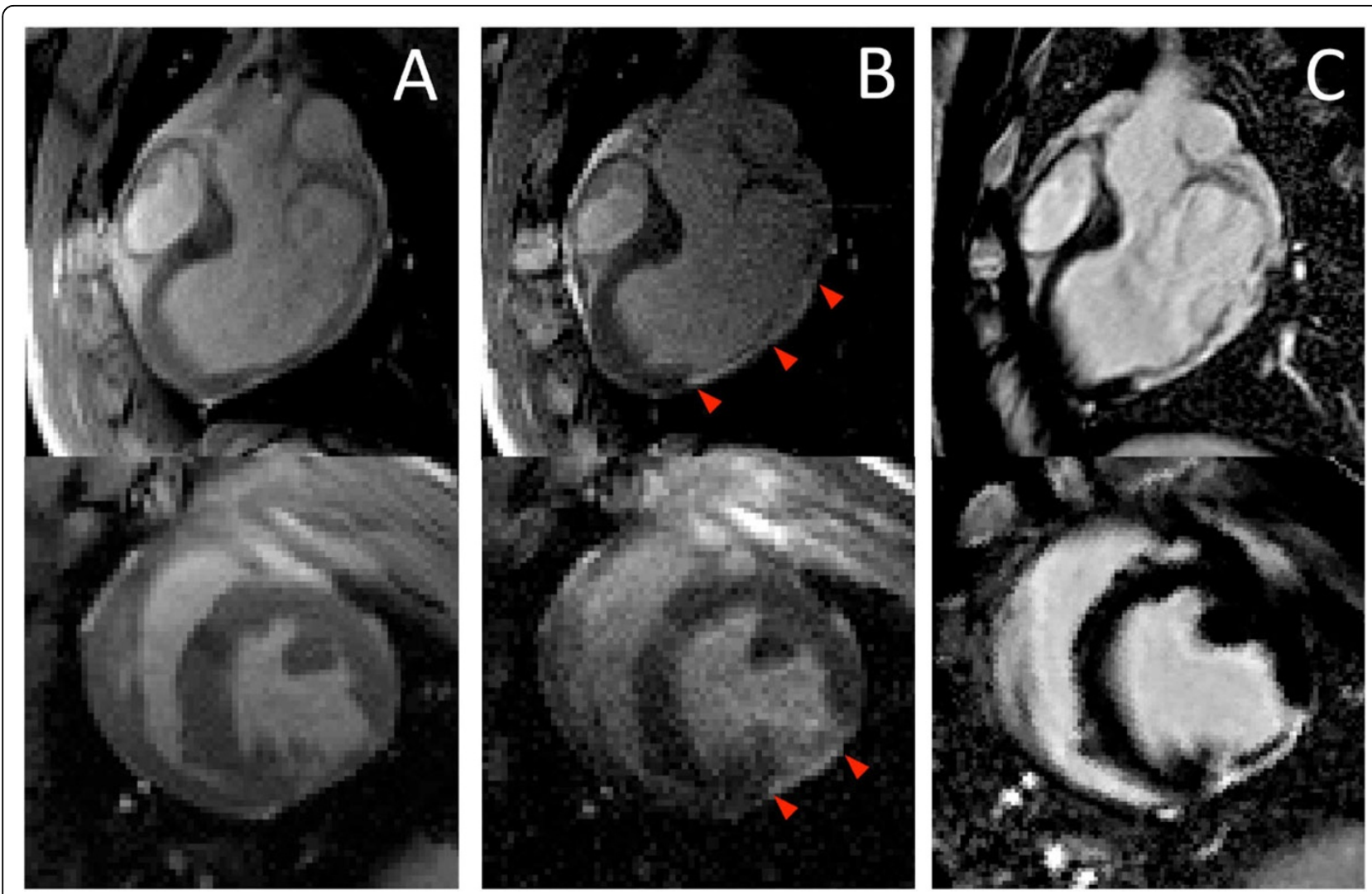

Figure 1 Comparison of standard real-time bSSFP images ("navigation mode") (A), real-time inversion recovery images ("infarct visualization mode") (B) and high-resolution ECG-gated breath-held LGE images (C) in two planes. Red arrowheads (B) indicate improved real-time infarct visualization compared to standard real-time imaging (A), and accurate infarct delieation compared to gold-standard late gadolinium enhancement images (C). 